International Mathematical Forum, 1, 2006, no. 28, 1359 - 1370

\title{
Easy Computations to Solution of Linear Fredholm Integral Equations
}

\author{
A. GOLBABAI and B. KERAMATI \\ Department of Applied Mathematics \\ Iran University of Science and Technology \\ Narmak, Tehran 16844, Iran \\ golbabai@iust.ac.ir
}

\begin{abstract}
In this paper we use Adomian's decomposition method to approximate the solution of linear Fredholm integral equation of the second kind with degenerate kernels and generating it to some nondegerate ones. The convergent of the method is also considered. Some examples are also given to show the efficiency of the method.
\end{abstract}

Keywords: Adomian's decomposition methold; Fredholm integral equations

Mathematics Subject Classification: 45A05

\section{INTRODUCTION}

Adomian's decomposotion method has been acclaimed as a significant power of method for solving integral equations $[1,2]$. In Fredholm integral equation of the second kind

$$
u(x)=f(x)+\lambda \int_{a}^{b} k(x, t) u(t) d t
$$


by using Adomian's method we take $u(x)=\sum_{n=0}^{\infty} u_{n}(x)$. So (1) can be written as follows:

$$
\begin{aligned}
u(x)= & f(x)+\lambda \int_{a}^{b} k(x, t) \cdot\left(\sum_{n=0}^{\infty} u_{n}(t)\right) d t \\
= & f(x)+\lambda \int_{a}^{b} k(x, t) u_{0}(t) d t+\lambda \int_{a}^{b} k(x, t) u_{1}(t) d t+\cdots \\
& +\lambda \int_{a}^{b} k(x, t) u_{n}(t) d t+\cdots
\end{aligned}
$$

Let $u_{0}=f(x)$, so we can write

$$
u_{n+1}(x)=\lambda \int_{a}^{b} k(x, t) u_{n}(t) d t \quad n=0,1,2, \ldots .
$$

By calculating the integrals, say up to $m$-terms, we can give an approximation $u^{(m+1)}(x)=\sum_{i=0}^{m} u_{n}(x)$ to the true solution $u(x)$.

For complicated kernels, this would be tedious and time consuming.

\section{KERNELS OF THE FORM $k(x, t)=g(x) \cdot h(t)$}

For integral equation

$$
u(x)=f(x)+\lambda \int_{a}^{b} g(x) h(t) \cdot u(t) d t
$$

we show that Adomian's method is an efficient method for obtaining an approximation to solution $u(x)$. We need only two integral evaluations, and for next steps no more integration is required.

We substitute $u(x)=\sum_{n=0}^{\infty} u_{n}(x)$ in $(3)$

$$
\begin{aligned}
u(x) & =f(x)+\lambda g(x) \int_{a}^{b} h(t) u_{0}(t) d t+\lambda g(x) \int_{a}^{b} h(t) u_{1}(t) d t+\cdots \\
u_{1}(x) & =\lambda g(x) \int_{a}^{b} h(t) u_{0}(t) d t=\lambda g(x) \int_{a}^{b} h(t) f(t) d t .
\end{aligned}
$$

Let $\alpha=\int_{a}^{b} h(t) f(t) d t$, then $u_{1}(x)=\alpha \lambda g(x)$. For $u_{2}(x)$ we have

$$
u_{2}(x)=\lambda g(x) \int_{a}^{b} h(t) u_{1}(t) d t=\lambda^{2} \alpha g(x) \int_{a}^{b} h(t) g(t) d t .
$$

Let $\beta=\int_{a}^{b} h(t) g(t) d t$, then $u_{2}(x)=\lambda \alpha \beta g(x)$. Thus $u_{3}(x)=\lambda^{3} \alpha \beta^{2} g(x)$, and in general

$$
u_{n}(t)=\lambda^{n} \alpha \beta^{n-1} \cdot g(x) .
$$


Hence the solution $u(x)=\sum_{n=0}^{\infty} u_{n}(x)$ can be written as follows

$$
u(x)=f(x)+\sum_{n=1}^{\infty} \alpha \beta^{n-1} \lambda^{n} g(x)
$$

or $u(x)=f(x)+C g(x)$, where

$$
C=\lambda \alpha\left[1+\lambda \beta+(\lambda \beta)^{2}+\cdots \cdot\right]
$$

If the integrals in $\alpha$ and $\beta$ are complicated, an efficient numerical method can be used to give good approximations to them.

Example 1. Approximate the solution of

$$
u(x)=\frac{9}{10} x^{2}+\int_{0}^{1} \frac{1}{2} x^{2} t^{2} u(t) d t .
$$

In this example $\lambda=1, u_{0}=f(x)=\frac{9}{10} x^{2}, g(x)=\frac{1}{2} x^{2}$ and $h(t)=t^{2}$, so we have

$$
\begin{aligned}
& \alpha=\int_{0}^{1} h(t) f(t) d t=\frac{9}{10} \int_{0}^{1} t^{4} d t=\frac{9}{50} \\
& \beta=\int_{0}^{1} h(t) g(t) d t=\frac{1}{2} \int_{0}^{1} t^{4}=\frac{1}{10} .
\end{aligned}
$$

By (4) we have

$$
u(x)=\frac{9}{10} x^{2}+\frac{9}{50}\left(1+\frac{1}{10}+\frac{1}{100}+\cdots\right)\left(\frac{1}{2} x^{2}\right)
$$

$u(x)=x^{2}$, which is in fact the exact solution.

\section{KERNELS OF THE FORM $k(x, t)=\sum_{i=1}^{N} g_{i}(x) h_{i}(x)$}

This is the main topic that we cover. In this section we derive an explicit formula for computing the terms $u_{n}(x)$. By substituting $k(x, t)$ in (1) we have

$$
\begin{aligned}
& u(x)=f(x)+\lambda \int_{a}^{b}\left(\sum_{k=1}^{N} g_{k}(x) h_{k}(t)\right) u(t) d t \\
& u(x)=f(x)+\lambda \sum_{k=1}^{N} g_{k}(x) \int_{a}^{b} h_{k}(t) \cdot u(t) d t
\end{aligned}
$$


by substituting $u(t)=\sum_{n=0}^{\infty} u_{n}(t)$ in (5) we have

$$
\begin{aligned}
u(x)= & f(x)+\lambda \sum_{k=1}^{N} g_{k}(x)\left[\int_{a}^{b} h_{k}(t)\left(\sum u_{n}(t) d t\right) \cdot d t\right] \\
u(x)= & f(x)+\lambda \sum_{k=1}^{N} g_{k}(x) \int_{a}^{b} h_{k}(t) u_{0}(t) d t \\
& +\lambda \sum_{k=1}^{N} g_{k}(x) \int_{a}^{b} h_{k}(t) \cdot u_{1}(t) d t+\ldots \\
& +\lambda \sum_{k=1}^{N} g_{k}(x) \int_{a}^{b} h_{k}(t) \cdot u_{n}(t) d t+\ldots
\end{aligned}
$$

Hence we can write

$$
u_{0}(x)=f(x), \quad u_{n+1}(x)=\lambda \sum_{k=1}^{N}\left[g_{k}(x)\left(\int_{a}^{b} h_{k}(x) u_{n}(t) d t\right)\right] \quad n=0,1,2, \ldots
$$

Let $\alpha_{k n}=\int_{a}^{b} h_{k}(t) \cdot u_{n}(t) d t$, so we have

$$
u_{n+1}(x)=\lambda \sum_{k=1}^{N} \alpha_{k n} g_{k}(x), \quad n=0,1,2, \ldots .
$$

By multiplying both sides of $(6)$ by $h_{m}(x) m=1,2, \ldots, N$ and then integrating on $[a, b]$ we obtain

$$
\int_{a}^{b} h_{m}(x) u_{n+1}(x) d x=\lambda \sum_{k=1}^{N} \alpha_{k n} \int_{a}^{b} h_{m}(t) g_{k}(t) d t, \quad m=1,2, \ldots, N .
$$

Now let

$$
\beta_{m k}=\int_{a}^{b} h_{m}(t) g_{k}(t) d t, \quad \alpha_{m, n+1}=\int_{a}^{b} h_{m}(t) u_{n+1}(t) d t .
$$

So (7) becomes

$$
\alpha_{m, n+1}=\lambda \sum_{k=1}^{N} \beta_{m k} \alpha_{k n}, \quad m=1,2, \ldots, N, n=0,1,2, \ldots
$$


We define the vector $\underline{\alpha}_{n}$ as

$$
\underline{\alpha}_{n}^{T}=\lambda\left[\alpha_{1 n}, \alpha_{2 n}, \ldots, \alpha_{N n}\right]
$$

and the matrix $B$ as

$$
B=\left[\beta_{i j}\right] \quad i=1,2, \ldots, N, \quad j=1,2, \ldots, N .
$$

The equations (8) now can be written as follows

$$
\underline{\alpha}_{n+1}=B \underline{\alpha}_{n}
$$

If we return to equations (6) we can write

$$
u_{n+1}=\underline{\alpha}_{n}^{T} \underline{g}(x)
$$

where $\underline{g}^{T}(x)=\left[g_{1}(x), g_{2}(x), \ldots, g_{N}(x)\right]$.

Now we formulate our method for approximating the solution $u(x)$ of integral equation (1) as following

$$
u_{0}(x)=f(x), \quad u_{n+1}(x)=\underline{\alpha}_{n}^{T} \cdot \underline{g}(x) \quad n=0,1,2, \ldots
$$

where

$$
\begin{gathered}
\underline{\alpha}_{n+1}=B \underline{\alpha}_{n}(x) \\
B=\left[\beta_{i j}\right] \quad i=1,2, \ldots, N \\
\underline{g}(x)=\left[g_{j}(x)\right], \quad j=1,2, \ldots, N \\
\underline{\alpha}_{0}=\left[\alpha_{i 0}\right], \quad \alpha_{i 0}=\int_{a}^{b} h_{i}(t) f(t) d t \\
\beta_{i j}=\int_{a}^{b} h_{i}(t) g_{j}(t) d t .
\end{gathered}
$$

Example 2. Approximate the solution of following integral equation by the above method

$$
u(x)=x+\int_{0}^{1}\left(x t^{2}+x^{2} t\right) u(t) d t .
$$

We have $k(x, t)=x t^{2}+x^{2} t, \lambda=1, u_{0}=x$,

$k(x, t)=g_{1}(x) h_{1}(t)+g_{2}(x) h_{2}(t), \quad g_{1}(x)=x, g_{2}(x)=x^{2}, h_{1}(t)=t^{2}, h_{2}(t)=t$ 


$$
\begin{aligned}
\alpha_{10} & =\int_{0}^{1} h_{1} u_{0} d t=\int_{0}^{1} t^{3} d t=\frac{1}{4}, \alpha_{20}=\int_{0}^{1} h_{2} u_{0} d t=\frac{1}{3} \\
\beta_{11} & =\int_{0}^{1} h_{1} g_{1} d t=\int_{0}^{1} t^{3} d t=\frac{1}{4}, \quad \beta_{12}=\frac{1}{5}, \beta_{21}=\frac{1}{3}, \quad \beta_{22}=\frac{1}{4} \\
\underline{\alpha}_{n+1} & =B \underline{\alpha}_{n},\left[\begin{array}{l}
\alpha_{1, n+1} \\
\alpha_{2, n+1}
\end{array}\right]=\left[\begin{array}{cc}
\frac{1}{4} & \frac{1}{5} \\
\frac{1}{3} & \frac{1}{4}
\end{array}\right]\left[\begin{array}{l}
\alpha_{1 n} \\
\alpha_{2 n}
\end{array}\right] \\
\underline{u}_{n+1} & =\underline{\alpha}_{n}^{T} \underline{g}(x), \\
\underline{u}_{1} & =\underline{\alpha}_{0}^{T} \underline{g}=\left[\begin{array}{ll}
\frac{1}{4} & \frac{1}{3}
\end{array}\right]\left[\begin{array}{c}
x \\
x^{2}
\end{array}\right]=\frac{1}{4} x+\frac{1}{3} x^{2}, \\
\underline{\alpha}_{1} & =B \underline{\alpha}_{0},\left[\begin{array}{l}
\alpha_{11} \\
\alpha_{21}
\end{array}\right]=\left[\begin{array}{ll}
\frac{1}{4} & \frac{1}{5} \\
\frac{1}{3} & \frac{1}{4}
\end{array}\right]\left[\begin{array}{l}
\frac{1}{4} \\
\frac{1}{3}
\end{array}\right]=\left[\begin{array}{l}
0.1291667 \\
0.1666667
\end{array}\right] \\
\underline{u}_{2} & =\underline{\alpha}_{1}^{T} \underline{g}(x), \quad u_{2}=0.1291667 x+0.1666667 x^{2} .
\end{aligned}
$$

$u_{3}, u_{4}, \ldots, u_{7}$ have been calculated by the same way as follows

$$
\begin{aligned}
u_{3} & =0.065625 x+0.0847222 x^{2} \\
& \vdots \\
u_{7} & =0.0043781 x+0.0056521 x^{2} .
\end{aligned}
$$

Therefore

$$
u(x) \simeq u_{0}+u_{1}+\cdots+u_{7}=1.50808 x+0.6664 x^{2} .
$$

The exact solution is

$$
u(x)=1.51261 x+0.67222 x^{2} .
$$

Example 3. Give an approximation to the solution $u(x)$ of integral

$$
u(x)=201 x^{2}-80 x+52+\int_{0}^{1}\left(-3 x^{2} t+4 x t^{2}-t^{3}\right) u(t) d t
$$

we have $\lambda=1, u_{0}=f(x)=201 x^{2}-80 x+52$

$$
\begin{aligned}
& g_{1}=-3 x^{2}, g_{2}=4 x, g_{3}=-1, h_{1}=t, h_{2}=t^{2}, h_{3}=t^{3} \\
& \alpha_{10}=\int_{0}^{1} h_{1}(t) f(t) d t=\int_{0}^{1} t\left(201 t^{2}-80 t+52\right) d t=49.5833 \\
& \alpha_{20}=\int_{0}^{1} h_{2}(t) f(t) d t=37.5333, \quad \alpha_{30}=30.5
\end{aligned}
$$


Easy computations to solution of linear Fredholm ...

$$
\begin{aligned}
& \beta_{11}=\int_{0}^{1} h_{1}(t) g_{1}(t) d t=\int_{0}^{1}\left(-3 t^{3}\right) d t=\frac{-3}{4} \\
& \beta_{12}=\int_{0}^{1} h_{1}(t) g_{2}(t) d t=\frac{4}{3}, \quad \beta_{13}=\frac{-1}{2} \\
& \beta_{21}=-\frac{3}{5}, \quad \beta_{22}=1, \quad \beta_{23}=\frac{-1}{3} \\
& \beta_{31}=\frac{-1}{2}, \quad \beta_{32}=\frac{4}{5}, \quad \beta_{33}=\frac{-1}{4} .
\end{aligned}
$$

So we have

$$
\begin{aligned}
& {\left[\begin{array}{l}
\alpha_{1, n+1} \\
\alpha_{2, n+1} \\
\alpha_{3, n+1}
\end{array}\right]=\left[\begin{array}{ccc}
\frac{-3}{4} & \frac{4}{3} & \frac{-1}{2} \\
\frac{-3}{4} & 1 & \frac{-1}{3} \\
\frac{-1}{2} & \frac{4}{5} & \frac{-1}{4}
\end{array}\right]\left[\begin{array}{c}
\alpha_{1 n} \\
\alpha_{2 n} \\
\alpha_{3 n}
\end{array}\right]} \\
& u_{0}=201 x^{2}-80 x+52 \underline{\alpha}_{0}=\left[\begin{array}{c}
49.5833 \\
37.5333 \\
30.5
\end{array}\right], \underline{g}=\left[\begin{array}{c}
-3 x^{2} \\
4 x \\
-1
\end{array}\right] \\
& u_{1}=\underline{\alpha}_{0}^{T} \underline{g}(x) \\
& u_{1}=49.5833\left(-3 x^{2}\right)+37.5333(4 x)+30.5(-1) \\
& u_{2}=\underline{\alpha}_{1}^{T} \underline{g}(x), \underline{\alpha}_{1}=B \underline{\alpha}_{0}=\left[\begin{array}{ccc}
\frac{-3}{4} & \frac{4}{3} & \frac{-1}{2} \\
\frac{-3}{5} & 1 & \frac{-1}{3} \\
\frac{-1}{2} & \frac{4}{5} & \frac{-1}{4}
\end{array}\right]\left[\begin{array}{c}
49.5833 \\
34.5333 \\
30.5
\end{array}\right] .
\end{aligned}
$$

Thus

$$
u_{2}=7.179229 x^{2}-9.533383 x+2.39001 \text {. }
$$

Similarly we have

$$
\begin{aligned}
& u_{3}=0.563946 x^{2}-0.60332 x+0.112636, \\
& u_{4}=0.011407 x^{2}-0.001982 x-0.001486, \\
& u_{5}=-0.004344 x^{2}+0.005162 x-0.001133 .
\end{aligned}
$$

The approximation to $\mathrm{u}(\mathrm{x})$ up to six terms is

$$
u(x) \simeq u_{0}+u_{1}+u_{2}+u_{3}+u_{4}+u_{5}=60.00468 x^{2}+59.9945 x+24.0012 .
$$


The exact solution is

$$
u(x)=60 x^{2}+60 x+24
$$

\section{Application to nondegenerate Kernels}

Consider the integral equation

$$
u(x)=f(x)+\lambda \int_{a}^{b} k(x, t) u(t) d t
$$

$k(x, t)$ under certain condition can be written as a Taylor series about the point $t=\alpha_{0} \in[a, b]$ as follows

$$
k(x, t)=k\left(x, \alpha_{0}\right)+\left(t-\alpha_{0}\right)\left[\frac{\partial k(x, t)}{\partial t_{0}}\right]_{t=\alpha_{0}}+\frac{\left(t-\alpha_{0}\right)^{2}}{2 !}\left[\frac{\partial^{2} k(x, t)}{\partial t^{2}}\right]_{t=\alpha_{0}}+\cdots
$$

In this case let $g_{i}(x)=\frac{\lambda}{i !}\left(\frac{\partial^{i} k}{\partial t^{i}}\right)_{t=\alpha_{0}}$ and $h_{i}(t)=\left(t-\alpha_{0}\right)^{i}$ then the method (9) can be applied here to give an approximation $u^{[m]}(x)$ to $u(x)$. Consider the following example:

Example 4. Approximate the solution $u(x)$ of the integral equation

$$
u(x)=\sin (x)+\int_{0}^{1}(1-x \cos (x t)] u(t) d t
$$

we can write

$$
\cos (x t)=1-\frac{x^{2}}{2 !} t^{2}+\frac{x^{4}}{4 !} t^{4}-\cdots
$$

Hence

$$
\begin{aligned}
& u(x) \simeq \sin x+\int_{0}^{1}\left[1-x\left(1-\frac{x^{2}}{2 !} t^{2}+\frac{x^{4}}{4 !} t^{4}\right)\right] u(t) d t \\
& u(x) \simeq \sin x+\int_{0}^{1}\left[(1-x)+\left(\frac{x^{3}}{2 !}\right) t^{2}+\left(\frac{-x^{5}}{4 !}\right) t^{4}\right] u(t) d t
\end{aligned}
$$


Now we have $\lambda=1, u_{0}=\sin (x)$.

$$
\begin{aligned}
& g_{1}=1-x, \quad g_{2}=\frac{1}{2} x^{3}, \quad g_{3}=\frac{-1}{4 !} x^{5} \\
& h_{1}=1, \quad h_{2}=t^{2}, \quad h_{3}=t^{4} \\
& \alpha_{10}=\int_{0}^{1} h_{1}(t) f(t) d t=\int_{0}^{1} \sin (t) d t=0.459698, \\
& \alpha_{20}=\int_{0}^{1} t^{2} \sin (t) d t=-0.2232443, \\
& \alpha_{30}=0.1466500 \\
& \beta_{11}=\int_{0}^{1} h_{1}(t) g_{1}(t) d t=\int_{0}^{1}(1-t) d t=0.5 \\
& \beta_{12}=0.125, \quad \beta_{13}=-0.006944, \quad \beta_{21}=0.083333, \quad \beta_{22}=0.083333 \\
& \beta_{23}=-0.005208, \quad \beta_{31}=0.033333, \quad \beta_{32}=0.0625, \quad \beta_{33}=-0.0041667 .
\end{aligned}
$$

Using (9) we have

$$
\begin{gathered}
u_{0}=f(x), \quad u_{n+1}=\underline{\alpha}_{n}^{T} \underline{g}(x), \quad \underline{\alpha}_{n+1}=B \underline{\alpha}_{n} \\
{\left[\begin{array}{l}
\alpha_{1, n+1} \\
\alpha_{2, n+1} \\
\alpha_{3, n+1}
\end{array}\right]=\left[\begin{array}{ccc}
0.5 & 0.125 & -0.006944 \\
0.083333 & -0.083333 & 0.005208 \\
0.033333 & -0.0625 & -0.0041667
\end{array}\right]\left[\begin{array}{l}
\alpha_{1 n} \\
\alpha_{2 n} \\
\alpha_{3 n}
\end{array}\right]}
\end{gathered}
$$

$u_{1}, u_{2}, \ldots, u_{10}$ have been calculated as follows

$$
\begin{aligned}
u_{1}= & 0.459698(1-x)+0.111622 x^{3}-0.00611 x^{5}, \\
u_{2}= & 0.256736(1-x)+0.028074 x-0.001194 x^{5}, \\
& \vdots \\
u_{10}= & 0.001448(1-x)+0.000136 x^{3}-0.00000516 x^{5} .
\end{aligned}
$$

Then

$$
\begin{aligned}
& u(x) \simeq u_{0}+u_{1}+\cdots+u_{10} \\
& u(x) \simeq \sin x+0.996911(1-x)+0.166367 x^{3}-0.008322 x^{5} .
\end{aligned}
$$

The true solution is $u(x)=1$ [3]. Some numerical results are given in the following table 


$\begin{array}{llllll}\mathrm{x} & 0 & 0.25 & 0.5 & 0.75 & 1\end{array}$

$\begin{array}{llllll}\text { approximated value } & 0.996911 & 0.99768 & 0.9984 & 0.99908 & 0.99952\end{array}$

$\begin{array}{llllll}\text { true value } & 1 & 1 & 1 & 1 & 1\end{array}$

\section{Convergence of the method}

There are some papers considering the convergence of Adomian's decomposition method applied to integral equations [3-5].If equation (1) satisfies the necessary conditions, then for our method it remains to show that the sequence $u^{[m]}(x)=\sum_{n=p}^{m-1} u_{n}(x)$ satisfies a Cauchy sequence, which we prove it in the following theorem.

Theorem. The sequence $u^{[m]}(x)=\sum_{n=0}^{m-1} u_{n}(x)$ as an approximated solution to integral equation

$$
u(x)=f(x)+\lambda \int_{a}^{b}\left(\sum_{i=1}^{N} g_{i}(x) h_{i}(t)\right) u(t) d t
$$

is a Cauchy sequence.

Proof. We prove $\lim _{m \rightarrow \infty}\left(u^{[m+1]}-u^{[m]}\right)=0$. Let $m \geq 0$ then

$$
\begin{aligned}
\left|u^{[m+1]}-u^{[m]}\right| & =\left|\sum_{n=0}^{m} u_{n}-\sum_{n=0}^{m-1} u_{n}\right|=\left|u_{m}\right| \\
& =\left|\underline{\alpha}_{m-1}^{T} \underline{g}\right| \leq\left\|\underline{\alpha}_{m-1}\right\|_{2} \cdot \| \underline{g}_{2}
\end{aligned}
$$

since $\underline{\alpha}_{m}=B \underline{\alpha}_{m-1}$, then

$$
\begin{aligned}
& \underline{\alpha}_{m-1}=B \underline{\alpha}_{m-2}=B\left(B \underline{\alpha}_{m-3}\right)=B^{2}\left(B \underline{\alpha}_{m-4}\right)=\cdots \\
& \underline{\alpha}_{m-1}=B^{m-1} \underline{\alpha} .
\end{aligned}
$$

So we can write

$$
\left|u^{[m+1]}-u^{[m]}\right| \leq\left\|B^{m-1} \underline{\alpha}_{0}\right\|_{2} \cdot\|\underline{g}\|_{2} .
$$

Obviously we have

$$
\forall \underline{x} \in R^{n}, \quad\|\underline{x}\|_{\infty} \leq \sqrt{n} .\|\underline{x}\|_{\infty} .
$$

Hence

$$
\begin{aligned}
\left|u^{[m+1]}-u^{[m]}\right| & \leq\left(\sqrt{N}\left\|B^{m-1} \underline{\alpha}_{0}\right\|_{\infty}\right)\left(\sqrt{N}\|\underline{g}\|_{\infty}\right) \\
& \leq\left(N\left\|\underline{\alpha}_{0}\right\|_{\infty} \cdot\|\underline{g}\|_{\infty}\right) \cdot\|B\|_{\infty}^{m-1}
\end{aligned}
$$


Let $\gamma=\|B\|_{\infty}=\max _{i} \sum_{i}\left|\beta_{i j}\right|$, so

$$
\left|u^{[m+1]}-u^{[m]}\right| \leq\left(N\left\|\underline{\alpha}_{0}\right\|_{\infty}\|\underline{g}\|_{\infty}\right) \gamma^{m-1}
$$

Put $M=N\left\|\underline{\alpha}_{0}\right\|_{\infty} \cdot\|\underline{g}\|_{\infty}$, then

$$
\left|u^{[m+1]}-u^{[m]}\right| \leq M \gamma^{m-1}
$$

and two cases should be considered

(1) $\gamma<1$, then obviously we have

$$
\lim _{m \rightarrow \infty}\left(u^{[m+1]}-u^{[m]}\right)=0
$$

(2) $\gamma \geq 1$, in this case we rewrite the kernel $k(x, t)=\sum_{i=1}^{N} g_{i}(x) h_{i}(t)$ as follows

$$
k(x, t)=2 \gamma \sum_{i=1}^{N} g_{i}(x)\left[\frac{h_{i}(t)}{2 \gamma}\right] d t
$$

and the integral equation becomes

$$
u(x)=f(x)+(2 \lambda \gamma) \int_{a}^{b} \sum g_{i}(x)\left[\frac{1}{2 \gamma} h_{i}(t)\right] \cdot u(t) d t
$$

Now introduce $\beta_{i j}$ as follows

$$
\beta_{i j}=\int_{a}^{b} \frac{1}{2 \gamma} h_{i}(t) g(t) d t, \quad B=\left[\beta_{i j}\right]
$$

Hence for the new $B,\|B\|_{\infty} \leq \frac{1}{2}$.

\section{Conclusion}

It has been proved that Adomian's decomposition method applied to linear Fredholm integral equations of the second kind is an efficient method which in fact increases the speed of computations in most problems. In this paper we used Adomian's decomposition for degenerate kernels of the form $\sum_{i=1}^{N} g_{i}(x) h_{i}(t)$ and derived an algorithm to simplify the calculation the terms $u_{n}$ in $u=\sum_{n=0}^{\infty} u_{n}$ and generated it to nondegenerate ones. Also we stated a theorem with the proof for convergence of the method. The efficiency of the method is shown in some solved examples. 


\section{REFERENCES}

[1] G. Adomian, Solving Frontier Problems of Physics: The Decomposition Method, Kluwer, 1994.

[2] G. Adomian, Nonlinear Stocastic Systems Theory and Applications to Physics, Kluwer, 1989.

[3] K.Abbaoui, Y. Cherruault,Convergence of Adomian's method applied to non-linear equations,Math. Compact.Model. 20(9)(1994)69-73.

[4] G. Adomian,Convergent series solution of non-linear equations,J. Comput.Math.Anal.Appl. 91 (1)( 1983).

[5] Y. Cherruault,Convergence of Adomian's method,Kyberneters 8 (2) (1988) 31-38.

\section{Received: November 7, 2005}

